\title{
CONTROL ADAPTATIVO POR MODELO DE REFERENCIA CON PREDICTOR SMITH A PARTIR DE LA REGLA MIT PARA UNA MESA VIBRATORIA DE DOS GRADOS DE LIBERTAD
}

\author{
Rafael Augusto Núñez-Rodríguez ${ }^{1}$
}

1 Docente investigador. Grupo de Investigación en Control Avanzado GICAV. Unidades Tecnológicas de Santander. Correo electrónico: ing.rafaeln@gmail.com

Recibido: 25 de julio del 2013. Aprobado: 25 de octubre del 2013.

Cómo citar este artículo: R. A. Núñez-Rodríguez, "Control adaptativo por modelo de referencia con predictor Smith a partir de la regla MIT para una mesa vibratoria de dos grados de libertad". Ingeniería Solidaria, Vol. 9, No. 16, pp. 89-95, Dic., 2013.

Resumen. Las mesas vibratorias se han generalizado para el análisis de estructuras civiles, debido a que permiten estimar y analizar la respuesta dinámica de un modelo a escala ante un sismo real. Muchos de estos sistemas se construyen a partir de actuadores lineales que permiten aplicar técnicas de control clásico. En este artículo se muestra la implementación de un sistema de control de posición adaptativo por modelo de referencia (MRAC), a partir de la regla MIT con predictor Smith sobre una mesa vibradora de dos grados de libertad impulsados por un motor trifásico acoplado a un mecanismo biela-manivela, cuyas características no lineales con alto grado de incertidumbre y tiempo muerto hacen necesaria la implementación de técnicas de control avanzado. La regla MIT es el enfoque original para el control adaptable basado en el modelo de referencia, al mismo tiempo se implementa un esquema predictor Smith para compensar los efectos del retardo en la respuesta del sistema. El sistema de control es implementado sobre una plataforma de 32 bit de Microchip conectado a un host.

Palabras clave: control adaptativo, mecanismo biela-manivela, predictor Smith, regla MIT.

\section{Model Reference Adaptive Control with Smith Predictor Using the Mit Rule for a Two-Degree-of-Freedom Shake Table}

\begin{abstract}
Shake tables have generally been used for civil structure analysis, as they allow the user to estimate and analyze a scale model's dynamic response to a real earthquake. Many of these systems are built using linear actuators which allow the application of classic control techniques. This article details the implementation of a model reference adaptive control (MRAC) system using the MIT rule with Smith predictor on a twodegree-of-freedom shake table powered by a three-phase motor coupled to a cam and connecting rod mechanism. The non-linear characteristics of this equipment, and the high degree of uncertainty and dead time, require the use of advanced control techniques. The MIT rule is the original focus for the MRAC, and a Smith predictor scheme is simultaneously used to compensate for system response delays. The control system is implemented on a 32-bit Microchip platform connected to a host.
\end{abstract}

Keywords: adaptive control, cam and connecting rod mechanism, Smith predictor, MIT rule.

\section{CONTROLE ADAPTATIVO POR MODELO DE REFERÊNCIA COM PREDITOR DE SMITH A PARTIR DA REGRA MIT PARA UMA MESA VIBRATÓRIA DE DOIS GRAUS DE LIBERDADE}

Resumo. As mesas vibratórias vêm se generalizando para a análise de estruturas civis, devido a que permitem estimar e analisar a resposta dinâmica de um modelo a escala ante um sismo real. Muitos desses sistemas se constroem a partir de atuadores lineares que permitem aplicar técnicas de controle clássico. Neste artigo, mostra-se a implementação de um sistema de controle de posição adaptativo por modelo de referência (MRAC), a partir da regra MIT com preditor de Smith sobre uma mesa vibradora de dois graus de liberdade impulsionados por um motor trifásico acoplado a um mecanismo biela-manivela, cujas características não lineares com alto grau de incerteza e tempo morto fazem necessária a implementação de técnicas de controle avançado. A regra MIT é o enfoque original para o controle adaptável baseado no modelo de referência, ao mesmo tempo se implementa um esquema preditor de Smith para compensar os efeitos do retardo na resposta do sistema. O sistema de controle é implementado sobre uma plataforma de 32 bit de Microchip ${ }^{\circ}$ conectado a um host.

Palavras-chave: controle adaptativo, mecanismo biela-manivela, preditor de Smith, regra MIT. 


\section{Introducción}

Las técnicas de control clásico son ampliamente utilizadas en procesos que tienen un alto grado de linealidad que permiten obtener un modelo matemático capaz de describir la dinámica del sistema. Sin embargo, cuando no existe certeza de los parámetros de la planta y sólo se conocen ciertas características dinámicas del modelo, y estos a su vez varían con el tiempo, se hace necesaria la aplicación de técnicas de control avanzado que no están ligadas directamente al modelo matemático del sistema que se desea controlar [1,2]. Los resultados de este trabajo de investigación están enfocados en este problema, ya que se requiere de un sistema de control para una mesa vibratoria que es utilizada para la evaluación de estructuras civiles a escala y sistemas de control antisísmicos activos. Cada eje de la mesa está impulsado por un motor de inducción trifásico acoplado a un mecanismo biela-manivela. Las señales de control generadas se aplican a la variante análoga de los variadores de frecuencia que regulan la velocidad de cada motor. $\mathrm{Al}$ acoplar todos estos dispositivos y mecanismos que constituyen la mesa, se observa que el comportamiento de esta en todo su rango dinámico no constituye un sistema lineal, lo que lleva a que el modelamiento matemático de la misma sea complejo con alto grado de incertidumbre, además del tiempo muerto propio de la naturaleza mecánica del sistema.

De esta manera, la técnica de control adaptativo se presenta como una buena alternativa para controlar este tipo de sistema, ya que permite aminorar los efectos de las variaciones de los parámetros de la planta; de igual forma, se introduce en el lazo de control un compensador de retardo a partir del predictor Smith que mitiga los efectos del tiempo muerto sobre la respuesta general del proceso. La combinación de estas dos estrategias se muestra como la técnica más adecuada para la generación de señales sísmicas en la mesa vibratoria, por su buen desempeño en sistemas con retardo en los que no se conoce completamente su modelo, a fin de obtener una buena aproximación en la reproducción de señales sísmicas. El sistema de control propuesto es un control de posición adaptativo por modelo de referencia por medio de la regla MIT con predictor Smith.

\section{Metodología}

Para el proceso de diseño del algoritmo de control adaptativo por modelo de referencia fue necesario preciar un modelo matemático de cada eje de la mesa vibratoria, el cual permitió estimar los parámetros del sistema y el tiempo muerto, factor importante en el momento de implementar el predictor Smith, para su posterior implementación hardware.

\subsection{Identificación del modelo matemático del sistema}

Habitualmente las mesas vibratorias están constituidas por un servo sistema hidráulico con características lineales, actuadores de la misma naturaleza que generan el desplazamiento lineal en cada eje y sensores para la realimentación del sistema de control [3-6].

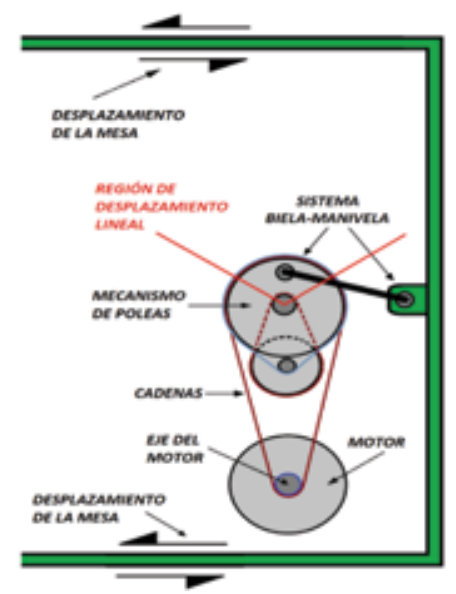

Figura 1. Mecanismo biela-manivela

Fuente: los autores

En este caso, el desplazamiento lineal del eje $\mathrm{X}$ y el eje Y son estructuras mecánicas basadas en un mecanismo biela-manivela impulsado por un motor inducción trifásico a través de una caja reductora compuesta por un sistema de cadenas y poleas dentadas, que en conjunto son la principal fuente incertidumbres y no linealidad del sistema, tal como se muestra en la figura 1.

Para obtener las funciones de trasferencia de cada eje, se modeló el sistema en la región de desplazamiento lineal que se muestra en la figura 1 , la cual permite un desplazamiento máximo $60 \mathrm{~mm}$, a $-60 \mathrm{~mm}$; los modelos matemáticos se obtuvieron utilizando técnicas de identificación tipo caja negra o Black Box utilizando la toobox de identificación de sistemas ident de MATLAB ${ }^{\oplus}$, con la que se consiguieron aproximaciones superiores al $90 \%$ en los dos modelos, incluyendo el retardo propio del sistema mecánico.

La aproximación del modelo lineal obtenido a partir de la identificación del sistema se muestra en la ecuación (1) para el eje X y la ecuación 2 para el eje 
Y, con los que se tienen aproximaciones del 91,8\% y $91,97 \%$, respectivamente [7].

$$
\begin{gathered}
G_{x}(s)=\frac{82,739 e^{-0,075121 s}}{s\left(0,0008484 s^{2}+0,02337 s+1\right)} \\
G_{y}(s)=\frac{77,977 e^{-0,077277 s}}{s(0,02136 s+1)}
\end{gathered}
$$

La validación de los resultados de los modelos de aproximación de la planta para cada eje se presentan en la figura 2, en la que las unidades de amplitud son voltios para la señal de entrada (señal cuadrada) y desplazamiento lineal en milímetros para las salidas simuladas y reales de cada eje (formas triangulares).

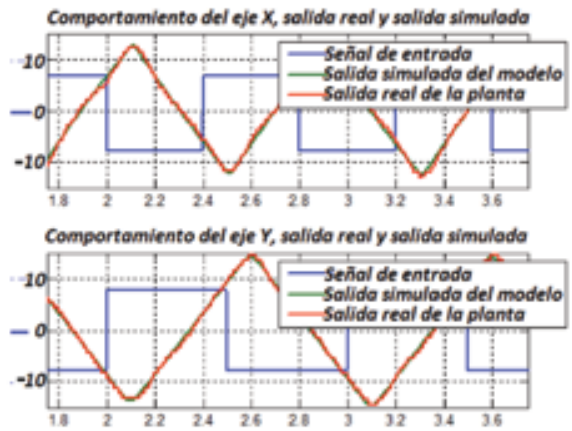

Figura 2. Resultados validación modelos Eje X y eje Y Fuente: los autores

\subsection{Diseño control adaptativo por modelo de referencia}

El objetivo del control adaptativo por modelo de referencia (MRAC) es hacer que el lazo de control tenga el mismo comportamiento dinámico de otro modelo que representa el comportamiento dinámico deseado del sistema en lazo cerrado [8]. En la figura 3 se observa el esquema de esta estrategia de control, donde se muestra claramente el mecanismo de adaptación, el lazo de realimentación negativa, el compensador de retardo a partir del predictor Smith, el lazo de control o ley de control con parámetro variables y el modelo de referencia en paralelo a todo el sistema.

La respuesta del modelo de referencia a una entrada representa la respuesta ideal que la planta debería tener en lazo cerrado. De acuerdo con esto, el controlador se debe diseñar de tal manera que la respuesta del lazo de control sea igual a la del modelo de referencia de tal forma que la diferencia entre la salida real del sis- tema y la salida del modelo de referencia tienda a cero, es decir, el mecanismo de adaptación ajusta los parámetros del controlador de tal manera que el sistema siga al modelo de referencia. En este trabajo, el mecanismo de adaptación está basado en la regla MIT.

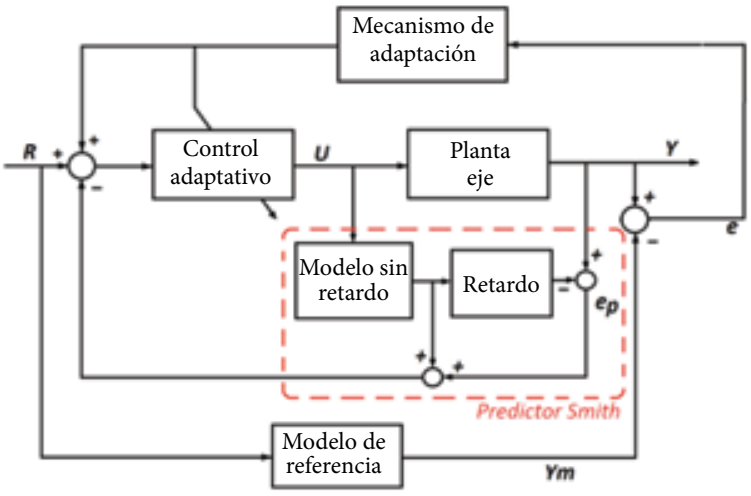

Figura 3. Diagrama de bloques sistema de control adaptativo por modelo de referencia

Fuente: los autores

\subsubsection{Modelo de referencia para el eje $X$ y eje $Y$}

La selección del modelo de referencia es un factor crítico al momento de diseñar la estrategia de control adaptativo, ya que este debe estar sujeto a la dinámica real de la planta en lazo cerrado; por lo tanto, es importante estimar un modelo matemático que a pesar de presentar incertidumbre, permita aproximar la dinámica de la planta para hacer una buena elección del modelo [9]. De esta manera se planteó un control de posición adaptativo con un parámetro de adaptación $\theta$ definido por la ley de control que se describe en la ecuación (3) [10].

$$
U=\theta_{j}^{T} \cdot w=\theta(R-Y)
$$

De la figura 3, la salida del proceso es el producto entre la ley de control $U(s)$ y el modelo de la planta $G p(s)$ en el dominio de la frecuencia. Dado que la salida del controlador está definida por la ecuación (3), y el modelo de la planta de cada eje con retardo puede ser aproximado a un modelo en lazo cerrado sin retardo en la trayectoria de realimentación gracias a la inclusión en el lazo de control del predictor Smith, tal como se propone en Normey, Rico y Camacho [11], se obtienen los modelos de referencia en lazo cerrado para el eje $\mathrm{X}$ y eje $\mathrm{Y}$, según las ecuaciones (4) y (5), a partir de las ecuaciones (1), (2) y (3). 


$$
\begin{gathered}
\frac{Y_{x}(s)}{R(s)}=\frac{\theta_{x} 82,739 e^{-0,075121 s}}{s\left(0,0008484 s^{2}+0,02337 s+1\right)+\theta_{x} 82,739} \\
\frac{Y_{y}(s)}{R(s)}=\frac{\theta_{y} 77,977 e^{0,077277 s}}{s(0,02136 s+1)+\theta_{y} 77,977}
\end{gathered}
$$

En las ecuaciones (4) y (5) se agregaron los retardos de cada modelo de la planta mostrado en las ecuaciones (1) y (2), y además se añadieron los valores apropiados para los parámetros $\theta_{x}$ y $\theta_{y}$, de manera que cada modelo de referencia describa adecuadamente la dinámica del sistema en lazo cerrado, haciendo que el modelo de referencia de cada eje tenga la respuesta deseada para una señal sísmica determinada estando acorde a la dinámica del sistema real. Según el criterio de diseño y analizando la respuesta del sistema real en lazo cerrado, se seleccionaron los valores de los parámetros $\theta_{x}$ y $\theta_{y}$ del modelo de referencia, en $00,5 \mathrm{y}$ 0,1 , respectivamente.

\subsubsection{Mecanismo de adaptación}

El mecanismo de adaptación se diseñó a partir de la regla mit desarrollada por el Instituto de Tecnología de Massachusetts, el cual implementa el método del gradiente para minimizar la función de costo dada por la ecuación (6), donde e es el error de seguimiento del sistema mostrado en la figura 3 y $\theta \theta$ representa los parámetros de la ley de control, el cual puede ser un escalar o un vector dependiendo de la cantidad de parámetros de la ley de control seleccionada $[7,11]$.

$$
J(\theta)=\frac{1}{2} e^{2}
$$

Para optimizar la función de costo, el parámetro $\theta$ debe cambiar en la dirección del gradiente negativo $J$ [9] dada por la ecuación (7), en la cual el parámetro $Y$ es la ganancia de adaptación, que debe ser seleccionada correctamente con el fin de reducir el tiempo de establecimiento y al mismo tiempo disminuir el transitorio del proceso de adaptación del sistema adaptativo [12].

$$
\frac{d \theta}{d t}=-y \frac{\partial J}{\partial \theta}=-y e \frac{\partial e}{\partial \theta}
$$

Para resolver la ecuación (7) es necesario calcular el gradiente del error de seguimiento a partir del modelo de referencia y el modelo matemático del sistema tal como lo muestra la ecuación (8).

$$
\frac{\partial e}{\partial \theta}=\frac{\partial(Y-Y m)}{\partial \theta}
$$

En donde el gradiente del término $\mathrm{Ym}$ es igual a cero, ya que el modelo de referencia no depende del parámetro $\theta$, de modo que el problema del cálculo del gradiente del error de seguimiento se reduce a la derivada parcial del modelo matemático de la planta en lazo cerrado respecto al parámetro de la ley de control $\theta$ [7], tal como se muestra en la ecuación (9) para el eje $\mathrm{X}$ y la ecuación (10) para el eje Y.

$$
\begin{gathered}
\frac{\partial e}{\partial \theta_{x}}=\frac{\partial\left(\frac{\theta_{x} 82,739 e^{-0,075121 s}}{s\left(0,0008484 s^{2}+0,02337 s+1\right)+\theta_{x} 82,739} \cdot R(s)\right)}{\partial \theta_{x}} \\
\frac{\partial e}{\partial \theta_{y}}=\frac{\partial\left(\frac{\theta_{y} 77,977 e^{-0,077277 s}}{s(0,02136 s+1)+\theta_{y} 77,977} \cdot R(s)\right)}{\partial \theta_{y}}
\end{gathered}
$$


Resolviendo las ecuaciones (9) y (10), y a partir de la ecuación (7), el mecanismo de adaptación del control adaptativo queda descrito por la ecuación (11) para el eje X y la ecuación (12) para el eje Y.

$$
\begin{gathered}
\theta_{x}=-\frac{y \cdot e \cdot(R(s)-Y(s))}{s}\left(\frac{82,73 e^{0,075 s}}{s\left(0,00084 s^{2}+0,023 s+1\right)+4,137}\right) \\
\theta_{y}=-\frac{y \cdot e \cdot(R(s)-Y(s))}{s}\left(\frac{77,97 e^{0,077 s}}{s(0,021 s+1)+3,89}\right)
\end{gathered}
$$

\section{Resultados}

Finalizado el diseño del algoritmo de control, a través del proceso de sintonización y validación por medio de simulaciones, el control adaptativo por modelo de referencia con predictor Smith, fue implementado sobre un sistema embebido basado en un microcontrolador de 32 bits PIC32 de Microchip sobre la plataforma Starter Kit del mismo fabricante. Las funciones de transferencia fueron convertidas a ecuaciones en diferencia en el dominio $\mathrm{Z}$ utilizando un retenedor de orden cero para su posterior programación sobre el controlador digital, con una frecuencia de muestreo de $200 \mathrm{~Hz}$, la cual se seleccionó de acuerdo con las señales sísmicas reales que se tomaron como referencia para reproducir sismos sobre la mesa.

En la figura 4 se observan los resultados de las pruebas del control adaptativo cuando la señal de entrada al sistema es una secuencia periódica de pulsos cuadrados. De acuerdo con esta figura, se pude observar que el sistema se adapta rápidamente siguiendo al modelo de referencia, con un gamma $Y$ de 0,0001 para el eje X y 0,0008 para el eje Y.
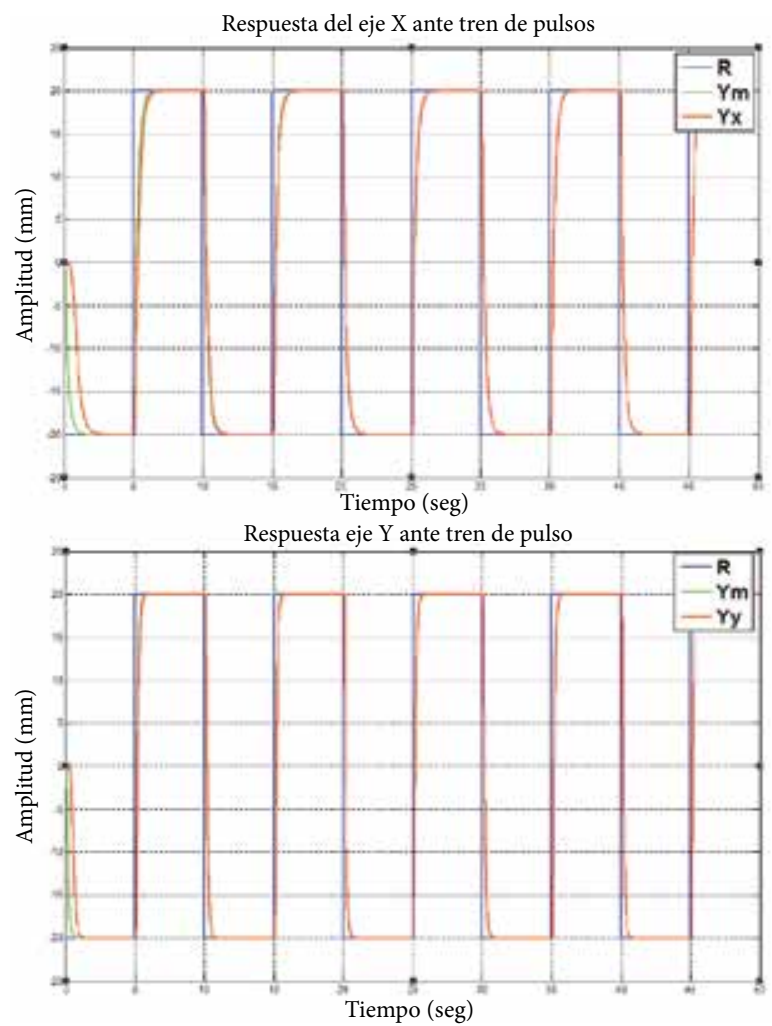

Figura 4. Respuesta eje $\mathrm{X}$ y eje $\mathrm{Y}$ ante tren de pulsos cuadrados Fuente: los autores 
Para el diseño del modelo de referencia se estableció el parámetro de la ley de control $\theta_{x}$ en 0,005 y $\theta_{y}$ en 0,1 , así que es de esperarse que los parámetros de adaptación converjan en estos valores, tal como lo muestra la figura 5, donde se observa claramente la convergencia y tiempo de convergencia de los parámetros ajustables $\theta_{x}$ y $\theta_{y}$ de la ley de control $U=\theta(R-Y) U=\theta(R-Y)$ para cada eje, los cuales son regidos por el mecanismo de adaptación. El parámetro $\theta_{x}$ converge en 0,005 y el parámetro $\theta_{y}$ converge en 0,1 , lo cual es uno de los indicadores de que el mecanismo funciona adecuadamente, haciendo que el sistema siga al modelo de referencia.
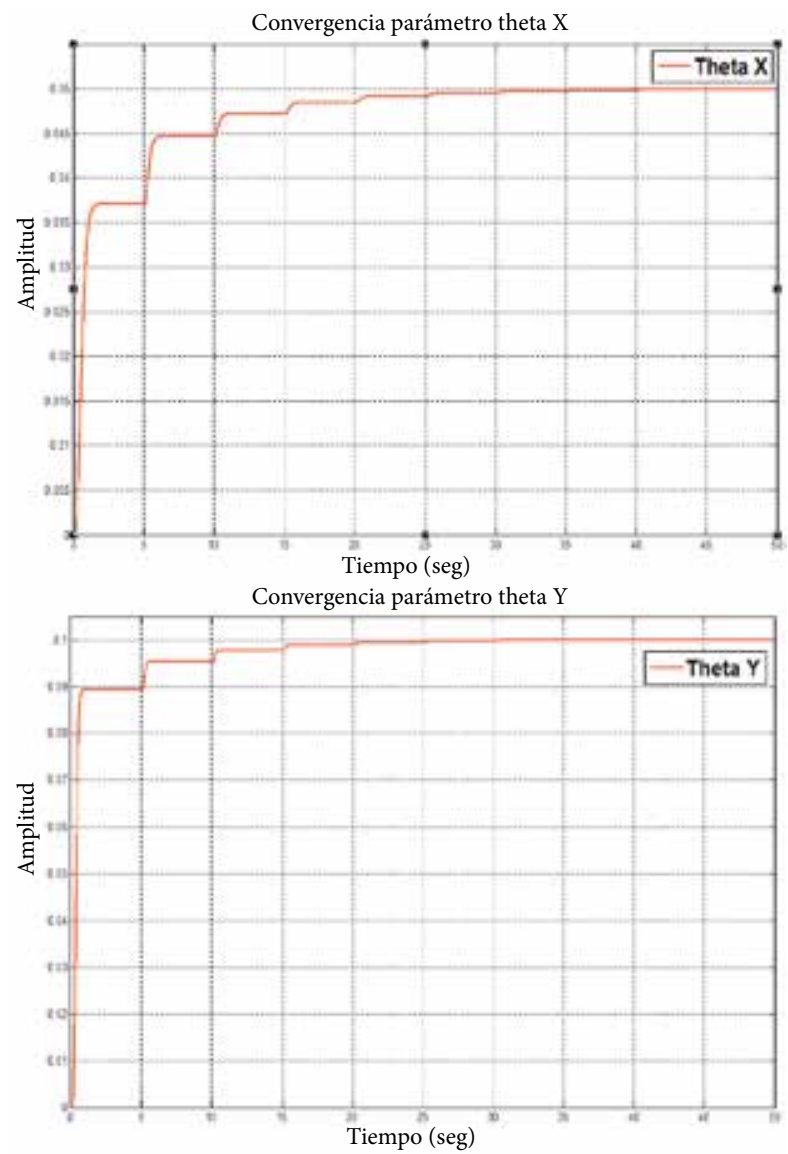

Figura 5. Convergencia parámetros de adaptación $\theta_{x}$ y $\theta_{y}$ Fuente: los autores

A partir de los resultados anteriores se realizaron pruebas de repetitividad ante señales sísmicas de referencia para la mesa vibradora. Los resultados presentados en la figura 6 muestran que el control adaptativo responde con un alto nivel de aproximación y repetitividad para las diferentes pruebas realizadas sobre cada eje de la mesa.

En este caso, para analizar la aproximación entre señal deseada dada por el modelo de referencia y la señal obtenida por la planta real, se optó por el cálculo del error medio cuadrático normalizado (NRMSE), dada en la ecuación (13) [13], y la medida del número de variabilidad entre las señales utilizando el coeficiente de determinación R2 descrito en la ecuación (14) [14].
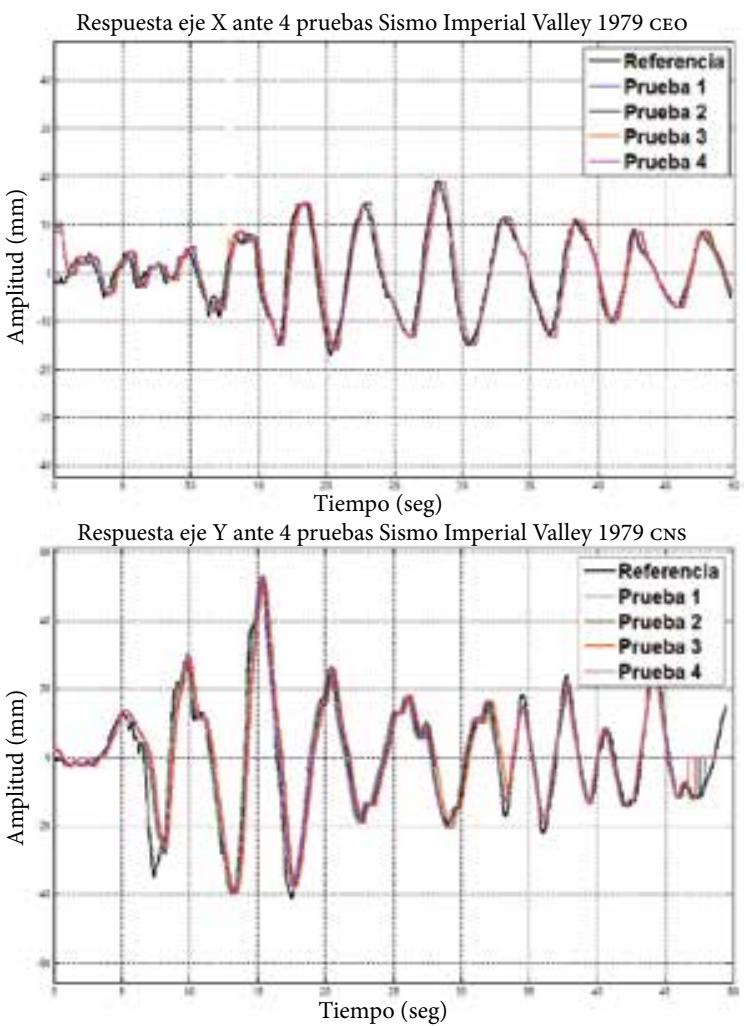

Figura 6. Respuesta control adaptativo ante señal Sismo Imperial Valley 1979

Fuente: los autores

$$
\begin{gathered}
R M S E=\sqrt{\frac{\sum_{i=1}^{n}\left(x_{i}-y_{i}\right)^{2}}{\sum_{i=1}^{n}\left(x_{i}\right)^{2}}} \\
R^{2}=1-\frac{\sum_{i=1}^{n}\left(x_{i}-y_{i}\right)^{2}}{\sum_{i=1}^{n}\left(x_{i}-\mu_{x i}\right)^{2}}=1-\frac{\operatorname{var}(e)}{\operatorname{var}\left(x_{i}\right)}
\end{gathered}
$$

La tabla 1 muestra los resultados obtenidos de las cuatro pruebas realizadas sobre la mesa vibratoria, tomando como señal de referencia una señal sísmica, cuyos datos fueron obtenidos del sismo ocurrido en Imperial Valley, CA, el 15 de octubre de 1979. Este sismo se seleccionó debido a que presenta un alto contenido de componentes de baja frecuencia acorde al ancho de banda del sistema, el cual está restringido por el retardo propio de la mesa, y fue descargado del Center of Engineering Strong Motion Data (CESMD). 
Tabla 1. NRSMSE y R2 para el eje X y eje $\mathrm{Y}$

\begin{tabular}{|l|c|c|c|c|}
\hline Test & NRMSE X & R2 X & NRMSE Y & R2 Y \\
\hline 1 & 0,24035 & 94,936 & 0,21373 & 97,412 \\
\hline 2 & 0,23405 & 95,919 & 0,19681 & 98,484 \\
\hline 3 & 0,25535 & 96,703 & 0,22321 & 98,913 \\
\hline 4 & 0,24085 & 95,411 & 0,20023 & 98,073 \\
\hline
\end{tabular}

Fuente: los autores

\section{Discusión y conclusiones}

Los resultados de esta investigación muestran que el sistema de control de una planta con alto grado de incertidumbre y con tiempo muerto puede ser implementado a partir de un sistema de control adaptativo por modelo de referencia mRac. Además fue posible implementar un algoritmo de control adaptativo con un parámetro de adaptación basado en la regla MIT con predictor Smith. Los sistemas inestables con retardo de tiempo se encuentran comúnmente en procesos de naturaleza mecánica y presentan complicaciones para su estudio, además de ser un reto para la estabilización del sistema. No obstante, es claro que el retardo de un sistema se puede dejar por fuera del lazo de realimentación [11], haciendo más fácil el análisis del sistema, obteniendo una respuesta similar al del sistema real, tal como se hizo al momento de determinar el modelo de referencia dejando el retardo del sistema por fuera de la ecuación característica [7], y con base en este modelo obtener la función de transferencia que rige la variación de cada uno de los parámetros de la ley de control.

El tiempo de convergencia de los parámetros $\theta_{x}$ y $\theta_{y}$ depende directamente de la ganancia de adaptación gamma $(\gamma)$, ya que de acuerdo con las ecuaciones (11) y (12), esta ganancia atenúa la salida de la función de transferencia de cada parámetro del controlador, influyendo directamente sobre la señal de control que a su vez determina el error de seguimiento del modelo de referencia, haciendo que el tiempo convergencia de los parámetros sea más lenta o más rápida, según el valor seleccionado para el gamma. Los sistemas de control adaptativo por modelo de referencia (MRAC) son una técnica de control avanzado no lineal [1]; por tanto, determinar el valor óptimo de la ganancia de adaptación es un factor crítico al momento del diseño de dicho algoritmo, ya que al ganar velocidad de convergencia de los parámetros de la ley de control, se puede reducir el margen de estabilidad pasando por un sistema oscilatorio hasta llegar a la inestabilidad de todo el sistema [12].

\section{Referencias}

[1] K. Benjelloun, H. Mechlih y E. Boukas, "A Modified Model Reference Adaptive Control Algorithm for DC Servomotor". Second IEEE Conference on Control Applications, Vancouver, B. C., Vol. 2, pp. 941-946, 1993.

[2] J. Tu, S. Jiang y D. Stoten, “The Seismic Response Reduction by Using Model Reference Adaptive Control Algorithm". Mechanic Automation and Control Engineering (MACE), pp. 1215-1218, 2010.

[3] J.Z. Chen, "Analysis of the Electro-hydraulic Servo Shaking Table with Flexible Payload". Technology, pp. 708-713, 2009.

[4] K. I. Seki, "Adaptive Compensation for Reaction Force With Frequency Variation in Shaking Table Systems". IEEE Transactions on Industrial Electronics, pp. 3864-3871, 2009.

[5] H. H. Gavin, "Control Objectives for Seismic Simulators". Control, 2009, pp. 3932-3937.

[6] K. I. Seki, "Reaction Force Compensation With Frequency Identifier in Shaking Table Systems". Control, 2010, pp. 673-678,

[7] C. Esparza, R. Núñez y F. González, "Model Reference Adaptive Position Controller with Smith Predictor for a Shaking-Table in two Axes". MICAI, 2013, pp. 271-282.

[8] F. R. Rubio y M. J. L. Sánchez, Control Adaptativo y Robusto, Sevilla: Secretariado de Publicaciones de la Universidad de Sevilla, 1996.

[9] K. Astrom y Wittenmark, Adaptive Control, Lund: Addison-Wesley Longman Publishing Co., 1994.

[10] P. Ioacnou, "Model Reference Adaptive Control". The Control Handbook, 2000, pp. 847-858.

[11] J. E. Normey y E. F. Camacho, "Predicción para control: una panorámica del control de procesos con retardo". Revista Iberoamericana de Automática e Informática Industrial, Vol. 3, No. 4, pp. 5-25, 2006.

[12] J. Stellet, "Influence of Adaptation Gain and Reference Model Parameters on System Performance for Model Reference Adaptive Control". World Academy of Science, Engineering and Technology, 2011, pp. 1768-1773.

[13] T. H. Frýza, "Video signals transparency in consequence of 3D-DCT transform”. Radioelektronika 2003 Conference Proceedings, 2003, pp. 127-130.

[14] D. R. Montgomery, Applied Statistics and Probability for Engineers, 3ra. ed. NY: John Wiley \& Sons, 2003. 